\title{
Pulmonary Embolism Mimicking Community-Acquired Pneumonia: A Case Series
}

\author{
Hakan Evren' ${ }^{(0}$, Emine Ünal Evren' $\odot$, Uğur Coșkun² ${ }^{\circledR}$ \\ 'Department of Infectious Diseases and Clinical Microbiology, University of Kyrenia, Kyrenia, Cyprus \\ ${ }^{2}$ Department of Cardiology, University of Kyrenia, Kyrenia, Cyprus
}

ORCID IDs of the authors: H.E. 0000-000I-8247-8I44; E.Ü.E. 0000-000I-9455-0473; U.C. 0000-0002-1958-7978.

Cite this article as: Evren H, Ünal Evren E, Coșkun U. Pulmonary Embolism Mimicking Community-Acquired Pneumonia: A Case Series. Cyprus J Med Sci 2019; 4(1): 60-2.

Pulmonary embolism (PE) is a severe medical condition that requires a high degree of clinical suspicion to prevent mortality and morbidity. PE can mimic pneumonia due to its clinical and laboratory findings. Here we report of three cases without an identifiable thrombotic source, who first presented with more likely pneumonia and were later diagnosed with and treated for PE.

Keywords: Pulmonary embolism, community-acquired pneumonia, lung

\section{INTRODUCTION}

Pulmonary embolism (PE) is a life-threatening clinical condition, and it is the third most common cause of cardiovascular death in the United States (I). Mortality is related to comorbidity factors and the time from diagnosis to treatment. When $P E$ is accompanied by pulmonary infiltrates in a chest $x$-ray, most of the pathologies in differential diagnosis include pneumonia, tuberculosis, pulmonary fungal disease, pulmonary vasculitis, etc. (2). Different clinical presentations make its diagnosing difficult (3). Community-acquired pneumonia (CAP) is one of the most common pulmonary syndromes, and it has similar symptoms to PE. A delayed PE diagnosis, due to clinical and radiological similarity with CAP, may cause increased mortality and morbidity. Herein, we report three cases of PE initially diagnosed and treated as CAP. Written informed consent was obtained from all patients.

\section{CASE PRESENTATIONS}

\section{Case I}

A 52-year-old male patient presented to the emergency department with a 3 days history of high fever, dry cough, and pleuritic right-sided chest pain. The patient had a fever of $38.8^{\circ} \mathrm{C}$, a heart rate of 108 beat/minute, an oxygen saturation of $95 \%$ (room air), and the blood pressure of $130 / 80 \mathrm{mmHg}$. A physical examination revealed decreased breath sounds in the right lower zone of the lung. Laboratory tests showed an increase in the white blood cell (WBC) count (I4,900 $\mu \mathrm{L})$ and C-reactive protein (CRP; $12.24 \mathrm{mg} / \mathrm{dL}$ ). A chest X-ray (CXR) showed the right lower lobe consolidation obscuring the right hemidiaphragm (Figure I). The patient was hospitalized with a diagnosis of CAP, and empirical antibiotherapy was initiated. The following day, he suffered from the exercise-induced shortness of breath. The oxygen saturation was $82 \%$ at room air. Subsequently, an echocardiography was performed by the cardiologist revealing a right heart chamber enlargement, right ventricular systolic disfunction, and an increased pulmonary artery pressure $(50 \mathrm{mmHg})$ that suggested pulmonary embolism. Diagnosis was confirmed by a computed tomography (CT) angiography of the thorax. As a result, thrombus formation was detected in the right pulmonary artery, predominantly on the bifurcation level. The lower limb Doppler ultrasound was performed, and it was negative for thrombus. We initiated low-molecule-weight heparin (LMWH) therapy. On the third day of hospitalization, patient's body temperature was normal, there was no dry cough, the CRP level significantly decreased ( $2.52 \mathrm{mg} / \mathrm{dL}$ ), and the sputum culture was negative. Thus, we excluded the CAP and discontinued the empirical antibiotic therapy. One week later, we switched the LMWH to Rivaroxaban 20 mg twice a day. On the I0th day of the anticoagulation therapy, the patient completely recovered. 


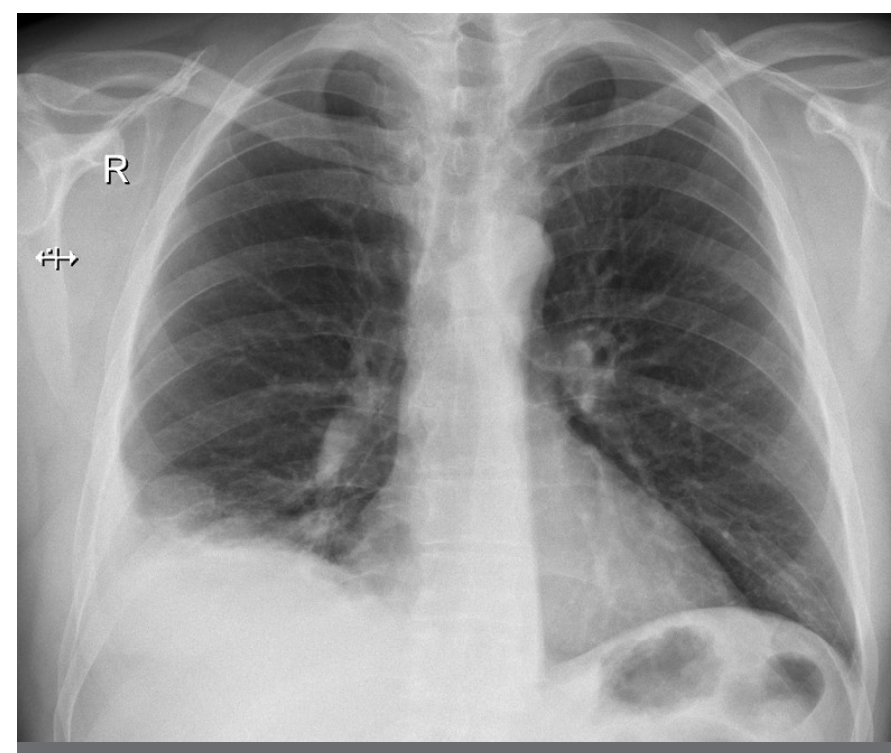

FIGURE I. Right lower lobe consolidation obscuring the right hemidiaphragm

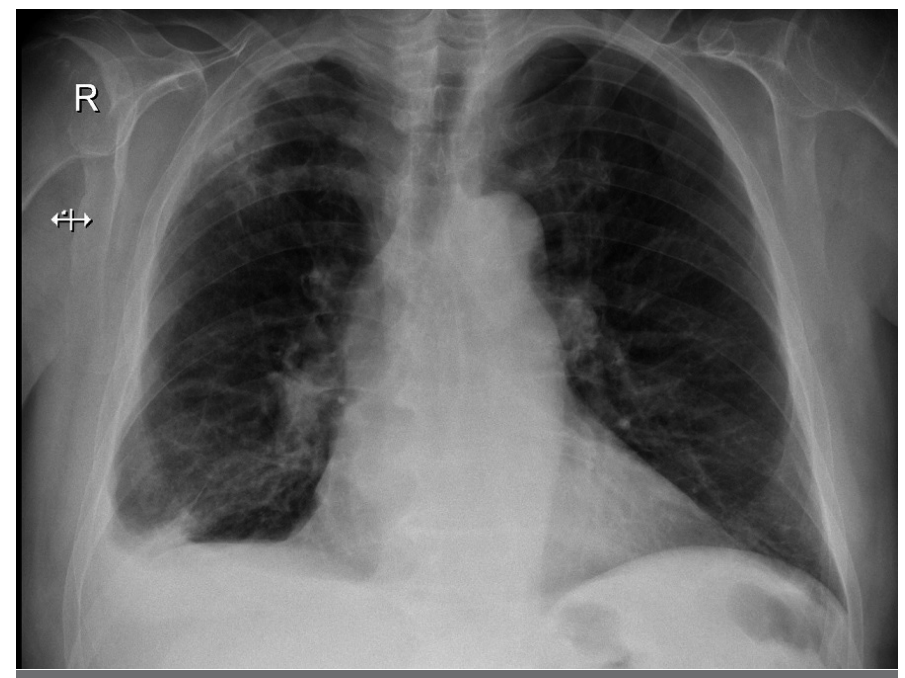

FIGURE 2. Right lower Lobe infiltration with effusion

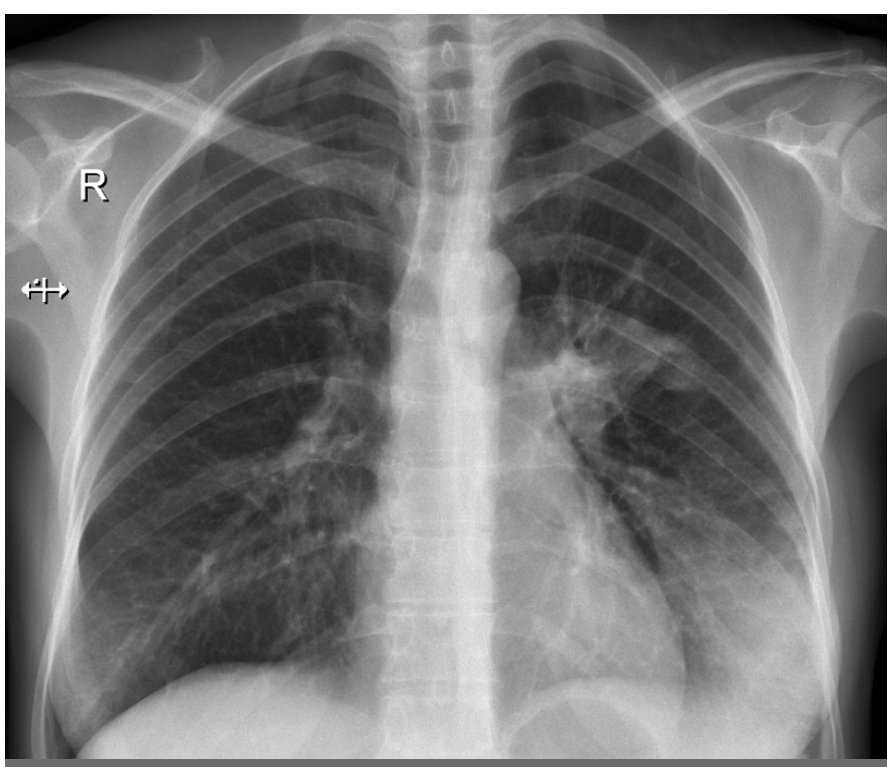

FIGURE 3. Left lower and mid-zones consolidation

\section{Case 2}

A 79-year-old male with a history of coronary artery disease presented to our clinic with complaints of high fever, cough, shortness of breath and back pain for 2 days. He was a smoker (30 packs a year, quit 20 years ago). The patient had a fever of $380^{\circ} \mathrm{C}$, a heart rate of 96 beats/minute, an oxygen saturation of $93 \%$ (room air), blood pressure of $145 / 85 \mathrm{~mm}$ of $\mathrm{Hg}$. The lung auscultation revealed rales in the right lower lung. Laboratory tests showed the WBC count of $18,400 \mu \mathrm{l}$ and CRP of $15.69 \mathrm{mg} /$ $\mathrm{dL}$. The CXR PA revealed an infiltration in the right lower lobe with effusion (Figure 2). The patient was initially treated with nonspecific antibiotherapy. Three days after the antibiotherapy, the patient suffered from sharp chest pain with worsening shortness of breath, and the SpO2 level was $82 \%$ at room air. The patient was evaluated at the cardiology clinic. Echocardiography revealed a severe right heart chamber enlargement, right ventricle basal segments hypokinesia, and a significantly increased pulmonary artery pressure. Diagnosis was confirmed by a CT angiography of the thorax, which revealed acute thrombus formation in the right main pulmonary artery. The LMWH was initiated. After the anticoagulation treatment, the patient was screened with echocardiography every day to see if there were any improvements in the right ventricular systolic function and pulmonary artery pressure. The patient continued with the LMWH and supportive treatment, he improved gradually within 5 days, and his pulse, respiratory rate, and sufficient oxygen saturation on room air values returned to normal.

\section{Case 3}

A 35-year-old female presented to our clinic with fever, cough, sputum, and hemoptysis that lasted for the past 4 days. She had small amounts of blood-streaked sputum. Her past medical history included asthma for 5 years. The patient had a fever of $38.1^{\circ} \mathrm{C}$, heart rate of 91 beats/minute, an oxygen saturation of $97 \%$ (room air) and blood pressure of $100 / 65 \mathrm{~mm}$ of $\mathrm{Hg}$. The lung auscultation revealed mild crackles in the left lower lung. Laboratory studies showed the WBC count at $12,700 \mu \mathrm{L}$ and CRP at $7,69 \mathrm{mg} / \mathrm{dL}$. The CXR PA showed an obvious infiltration and consolidation in the lower and mid-zone of the left lung (Figure 3).

Levofloxacin Ix500 mg tablets were prescribed. Five days after the visit, the patient presented to our emergency department with an abrupt onset of chest pain and significantly increased bloody sputum. To exclude other medical conditions, a cardiology consultation was requested. Echocardiography revealed a moderate right heart chamber enlargement, moderate right ventricle systolic dysfunction, and markedly increased pulmonary artery pressure. A CT angiography of the thorax was performed, and it revealed multifocal embolus in the posterobasal and laterobasal branches of the left pulmonary artery. The LMWH was initiated, and close monitoring of coagulation parameters and vital signs has been done. Within four days of the LMWH therapy, the oxygen saturation returned to normal.

\section{DISCUSSION}

Community-acquired pneumonia is an acute infection of the lung. It was reported that $5 \%-17 \%$ of patients with diagnosed pneumonia actually suffer from non-infectious CAP that is mimicked. In addition, CRP and procalcitonin may be elevated 
in many of such cases. Usually, CAP shows significant improvement in vital signs within 48-72 hours following the antibiotic therapy initiation (4). Even if there are different reasons of the CAP treatment failure, in this study, we focused on PE.

Clinical suspicion of PE is not a frequent consideration in patients who present with classical infectious diseases symptoms. Furthermore, the prevalence of CAP complicated with $P E$ is very rare (3). Cough, fever, dyspnea, sputum, and pleuritic pain are the main symptoms of CAP, as well as PE. Coexistence may also occur rarely. However, if physicians were aware of $\mathrm{PE}$, further investigation for diagnosis would be performed correctly (2).

Imaging tests are important in confirming the diagnosis. Contrast-enhanced computed tomography pulmonary angiography (CTPA) is the standard imaging modality for the diagnosis of PE. Ventilation/perfusion lung scanning is an alternative to CTPA and useful for patients in whom CTPA is contraindicated. However, it should be kept in mind that nearly two-thirds of the patients have a non-diagnostic result, which is neither normal nor high probability (4). There are many risk factors for $\mathrm{PE}$, such as recent surgery, pregnan$c y$, oral contraceptives, immobilization, and trauma (5). The only relative risk that we suspected was physical inactivity in the first case due to patient's profession. Other cases did not have any remarkable risk. All of these patients had negative hypercoagulability screen tests.

Pulmonary embolism is treated by anticoagulants in all patients who do not have a significant contraindication (I). If there is no response to anticoagulant therapy, pulmonary embolectomy surgery can be applied (6). In our cases, we started the LMWH treatment and continued with oral anticoagulants.

In conclusion, PE mimicking CAP is not uncommon. Therefore, clinicians must be aware of this medical condition, particularly in patients who do not respond to antibiotic treatment.

Informed Consent: Informed consent was obtained from the patients who participated in this study.

Peer-review: Externally peer-reviewed.

Author contributions: Concept - E.U.E., U.C.; Design - H.E., E.U.E.; Supervision - H.E., U.C.; Resource - H.E., E.U.E.; Materials - U.C., H.E.; Data Collection and/or Processing - H.E., E.U.E.; Analysis and/or Interpretation - U.C., E.U.E.; Literature Search - E.U.E., H.E.; Writing - H.E., E.U.E.; Critical Reviews - U.C., H.E.

Conflict of Interest: The authors have no conflicts of interest to declare.

Financial Disclosure: The authors declared that this study has received no financial support.

\section{REFERENCES}

I. Corrigan D, Prucnal C, Kabrhel C. Pulmonary embolism: the diagnosis, risk-stratification, treatment and disposition of emergency department patients. Clin Exp Emerg Med 2016; 3: I17-25. [CrossRef]

2. Xu K, Tang X, Song Y, Chen Z. The diagnostic dilemma between pulmonary embolism with positive chest imaging and pneumonia: a case report and literature review. J Transl Med Epidemiol 2015; 3: 1039.

3. Koc I. Pulmonary Embolism Mimicking Community Acquired Pneumoniað58; A Case Report. J Clin Anal Med 2016; 6: 662-4.

4. Black AD. Non-infectious mimics of community-acquired pneumonia. Pneumonia (Nathan) 2016; 8: 2. [CrossRef]

5. Rana S. Acute pulmonary embolism in young. J Assoc Chest Physicians 2017; 5: 46. [CrossRef]

6. Conkbayır C, Kenan S, Emiroglu O. Massive pulmonary thromboembolism after abdominoplasty and liposuction. Turk Kardiyol Dern Ars 20II; 39: 4I0-3. [CrossRef] 